\title{
Reply to "Differences in the Impact of Age on Mortality in Well-Differentiated Thyroid Cancer"
}

\author{
Norman G. Nicolson, MD and Tobias Carling, MD, PhD \\ Department of Surgery, Yale University School of Medicine, New Haven
}

To the editors:

We read with great interest the recent publication by Yan et al. ${ }^{1}$ highlighting the important prognostic differences between follicular thyroid cancer (FTC) and the far more commonly encountered papillary thyroid cancer (PTC). FTC recurs rarely, but patients who have recurrences may die of metastatic disease in spite of otherwise favorable risk profiles. The different biology between the categories of well-differentiated thyroid cancer (i.e., PTC and FTC) is evident both clinically and in the laboratory.

Furthermore, our group recently published a whole exome-sequencing analysis of FTC that mapped the genetic landscape of these tumors, with a particular emphasis on the infrequent widely invasive (WIFTC) subtype. $^{2}$ Of 10 WIFTC patients studied, 5 experienced recurrence and died of FTC within 10 years. Of 6 thyroid cancer-specific deaths in our overall FTC cohort of 39 cases, half were classified as stage I or II by the American Joint Committee on Cancer (AJCC) eighth-edition staging system, mostly due to the increased age cutoff of 55 years. ${ }^{3}$ Interestingly, we found that both genomic markers and traditional clinical and histopathologic factors predicted poor survival.

Unfortunately, the degree of FTC invasion is not wellcaptured in the national cancer registries, so a large-scale analysis of these high-risk subgroups is not possible using these approaches. Prior studies have demonstrated the prognostic significance of the degree of invasion for FTC in smaller single-institution series. ${ }^{4}$ Although the AJCC eighth-edition staging system provides accurate prognostic information for the vast majority of patients with welldifferentiated thyroid cancer, certain high-risk histologic or molecular features may outweigh traditional tumor-nodemetastasis (TNM) staging and should be considered when the risk of recurrence and death is evaluated for thyroid cancer patients.

\section{REFERENCES}

1. Yan $\mathrm{H}$, Winchester DJ, Prinz RA, Wang CH, Nakazato Y, MooYoung TA. Differences in the impact of age on mortality in welldifferentiated thyroid cancer. Ann Surg Oncol. 2018;25:3193-9.

2. Nicolson NG, Murtha TD, Dong W, et al. Comprehensive genetic analysis of follicular thyroid carcinoma predicts prognosis independent of histology. $J$ Clin Endocrinol Metab. 2018;103:2640-50.

3. Tuttle RM, Haugen B, Perrier ND. Updated American Joint Committee on Cancer/tumor-node-metastasis staging system for differentiated and anaplastic thyroid cancer (eighth edition): what changed and why? Thyroid. 2017;27:751-6.

4. Sugino K, Ito K, Nagahama M, et al. Prognosis and prognostic factors for distant metastases and tumor mortality in follicular thyroid carcinoma. Thyroid. 2011;21:751-7.
(C) Society of Surgical Oncology 2018

First Received: 17 October 2018;

Published Online: 12 December 2018

T. Carling, MD, PhD

e-mail: tobias.carling@yale.edu 